\title{
Leukocytoclastic Vasculitis as an Extraintestinal Manifestation of Crohn's Disease
}

\author{
Thiara Barcelos Rocha ${ }^{a} \quad$ Ana Lorena Sousa de Vasconcelos Garate ${ }^{a}$ \\ Rodrigo Fedatto Beraldo ${ }^{a}$ Sean Hideo Shirata Lanças ${ }^{a}$ \\ Fábio Vicente Leite ${ }^{a}$ Rodrigo Quera ${ }^{b}$ Jaqueline Ribeiro de Barros ${ }^{a}$ \\ Julio Pinheiro Baima ${ }^{a}$ Rogerio Saad-Hossne ${ }^{a} \quad$ Ligia Yukie Sassakia $^{a}$

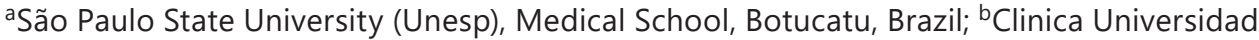 \\ de los Andes, Santiago, Chile
}

\section{Keywords}

Leukocytoclastic vasculitis - Extraintestinal manifestation · Crohn's disease · Inflammatory bowel disease . Case report

\begin{abstract}
Cutaneous involvement is one of the most common extraintestinal manifestations of inflammatory bowel disease (IBD). More commonly, pyoderma gangrenosum and erythema nodosum are noted, but psoriasis, aphthous stomatitis, Sweet's syndrome, and vasculitis may also occur. Leukocytoclastic vasculitis (LCV) is a rare cutaneous manifestation, characterized by the appearance of palpable purpura, urticaria, and ulcer-necrotic lesions predominantly in the lower extremities that improve with immunosuppressive therapy. In this case, we report a patient with CD and LCV. We also searched the literature on the diagnosis and treatment of LCV in patients with CD. Female, 31, presented with diarrhea containing mucus and blood, abdominal pain, arthralgia, and enanthematous plaques and ulcers with a hematinic background in the lower extremities. The results of the colonoscopy were compatible with $C D$ and skin biopsy showed signs of LCV. Systemic autoimmune disease and primary vasculitis were ruled out. The patient received treatment with a systemic corticosteroid and the skin lesions improved. Outpatient treatment with antitumor necrosis factor therapy was initiated to promote skin healing and IBD clinical remission. As LCV is a rare manifestation of IBD, it is necessary to distinguish this dermatopathy from other systemic vasculitis. The engagement of a multidisciplinary team is essential for the correct diagnosis and management.




\section{Introduction}

Inflammatory bowel disease (IBD) is a chronic disease resulting from the interaction between genetic and environmental factors, which can affect the entire gastrointestinal tract. IBD is subdivided into Crohn's disease and ulcerative colitis and is associated with several extraintestinal symptoms such as cutaneous, musculoskeletal, ophthalmological, and hepatobiliary manifestations [1-3], which occur in about $6-47 \%$ of individuals with the disease [4].

Cutaneous manifestations are one of the most common [5, 6], affecting up to $15 \%$ of individuals with IBD [7]. These usually appear within 2 years of follow-up [1] and are more prevalent in individuals with Crohn's disease (20-40\%) compared to those with ulcerative colitis (15-20\%) [8]. The most frequent cutaneous manifestations are erythema nodosum and pyoderma gangrenosum. However, psoriasis, aphthous stomatitis, Sweet's syndrome, and vasculitis can also occur [2, 3, 6-11].

Cutaneous vasculitis is considered a rare manifestation, but there are reports of its association with Crohn's disease $[5,9,11]$. The present study aims to describe the case of an individual with Crohn's disease who presented with leukocytoclastic vasculitis (LCV) and to summarize what is known from the literature regarding LCV diagnosis and treatment to elucidate clinical recommendations.

The study was approved by the local Research Ethics Committee (CAAE: 25857119.7.0000.5411). Written informed consent was obtained from the patient for publication of this case report and any accompanying images.

\section{Case Report/Case Presentation}

A 31-year-old female patient, Caucasian, nonsmoking, presented with a 4-year history of intermittent abdominal pain and diarrhea, but without systemic symptoms or repercussions to her daily routine. As part of the medical investigation, she underwent a colonoscopy in April 2018 which showed edema, enanthema, and erosion in the rectum, descending, sigmoid, and transverse colon, which are all signs of a mild-to-moderate inflammatory response in the colon. The anatomopathological exam showed edema and lymphoplasmic infiltrate with eosinophils and the presence of lymphoid follicles, alterations of the glandular architecture, and acute cryptitis, consistent with the diagnosis of ulcerative colitis. Treatment with oral mesalazine was prescribed, but the patient did not take the medication. However, she showed spontaneous improvement of the intestinal symptoms.

In March 2019, the patient was admitted to the hospital with diarrhea which was occurring $>10$ times a day. She presented with liquid stools containing mucus and blood, moderate abdominal pain, asthenia, weight loss, and fever with no improvement after antibiotics and antiparasitic treatment. In addition, she reported having arthralgia and painful lesions in her lower extremities. On physical examination, she was in regular condition, pale, dehydrated, and feverish. Her abdomen was flaccid and painful during deep palpation. The patient had multiple reddish-brown macular lesions in her lower extremities, and some were coalescing to form ulcerations (Fig. 1). Laboratory tests showed normocytic and normochromic anemia (hemoglobin: $11.4 \mathrm{~g} / \mathrm{dL}$ and hematocrit: $35.8 \%$ ), leukocytosis $\left(14.2 \times 10^{3}\right.$ per $\left.\mathrm{mm}^{3}\right)$, hypoalbuminemia $(2.6 \mathrm{~g} / \mathrm{dL})$ and a high concentration of high-sensitivity C-reactive protein $(21.2$ $\mathrm{mg} / \mathrm{dL}$, reference value $=1.0 \mathrm{mg} / \mathrm{dL}$ ). The colonoscopy at this time showed ulcerations in the terminal ileum and cecum, the ascending, transverse, descending, and sigmoid colon, rectum, and perianal fistulae compatible with Crohn's disease resulting in a Simple Endoscopic Score for Crohn Disease score of 15 points (Fig. 2). The histopathological exam showed erosion and lymphoid follicular hyperplasia in the terminal ileum and focal erosions in the colon

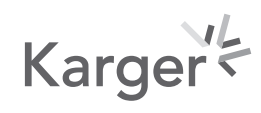



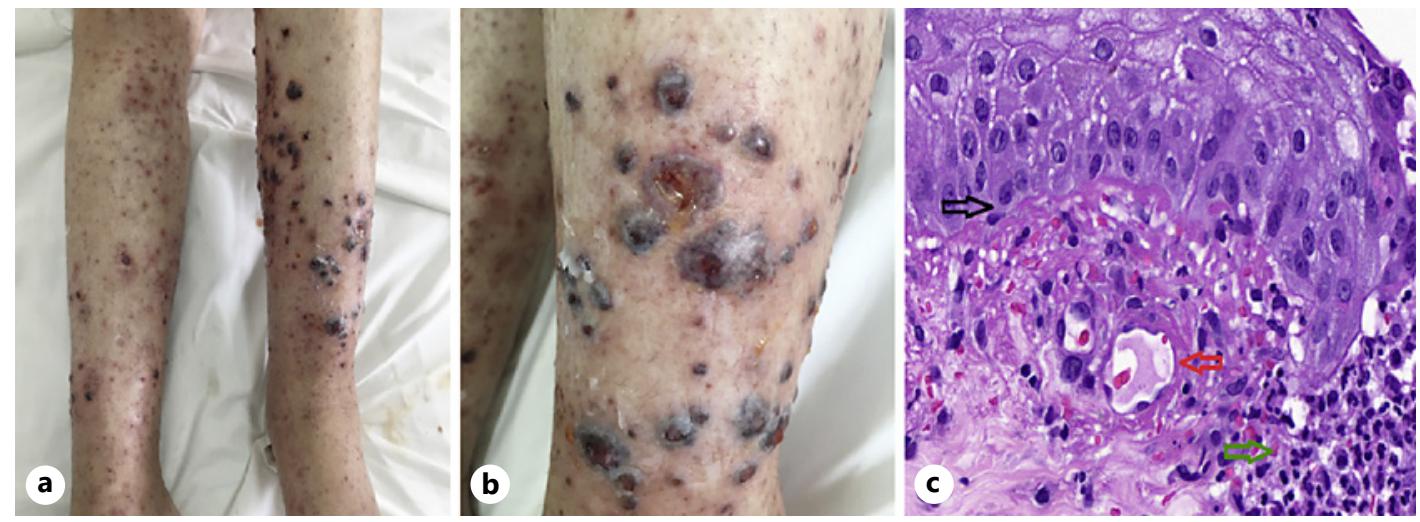

Fig. 1. Multiple reddish-brown macular lesions in the lower limbs (a), some coalescent forming ulcerations in the skin (b). Skin histopathology showing interface dermatitis (black arrow), fibrinoid necrosis with thickening of endothelium and fibrin area (red arrow) and dense neutrophil population (green arrow), suggestive of LCV. Magnification, $\times 400$ (c). LCV, leukocytoclastic vasculitis.

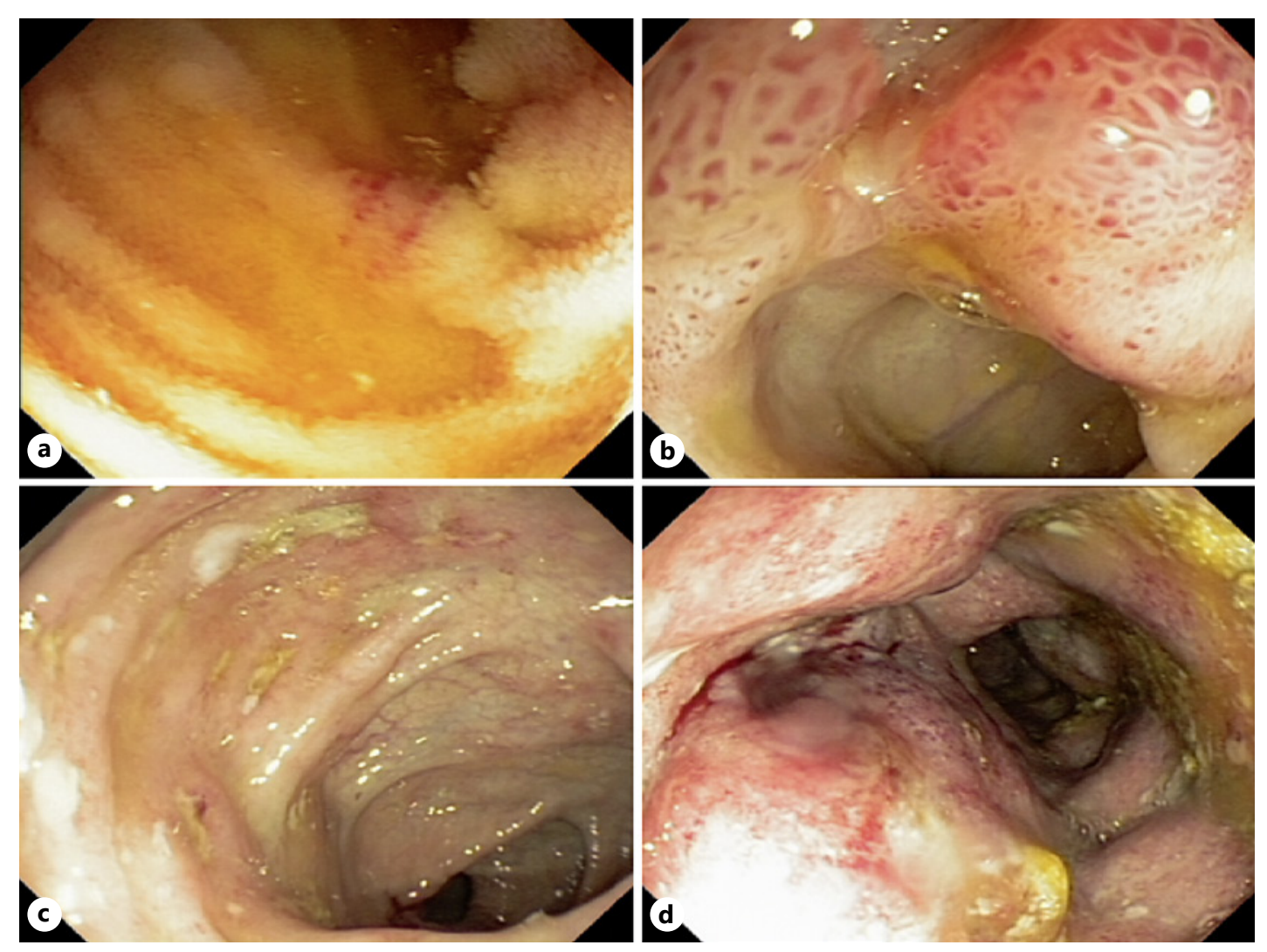

Fig. 2. Images of ileocolonoscopy performed during hospitalization (March 2019) showing ulcerations in the terminal ileum (a), cecum (b), ascending, transverse (c), descending (d), sigmoid, and rectum.

fragments. The focal and erosive character of the lesions indicated the possibility of Crohn's disease despite the absence of epithelioid microgranulomas. The abdominal and pelvic tomography showed a slight thickening of the small intestine, involving the jejunoileal segment and increased size and number of mesenteric and iliac lymph nodes. 


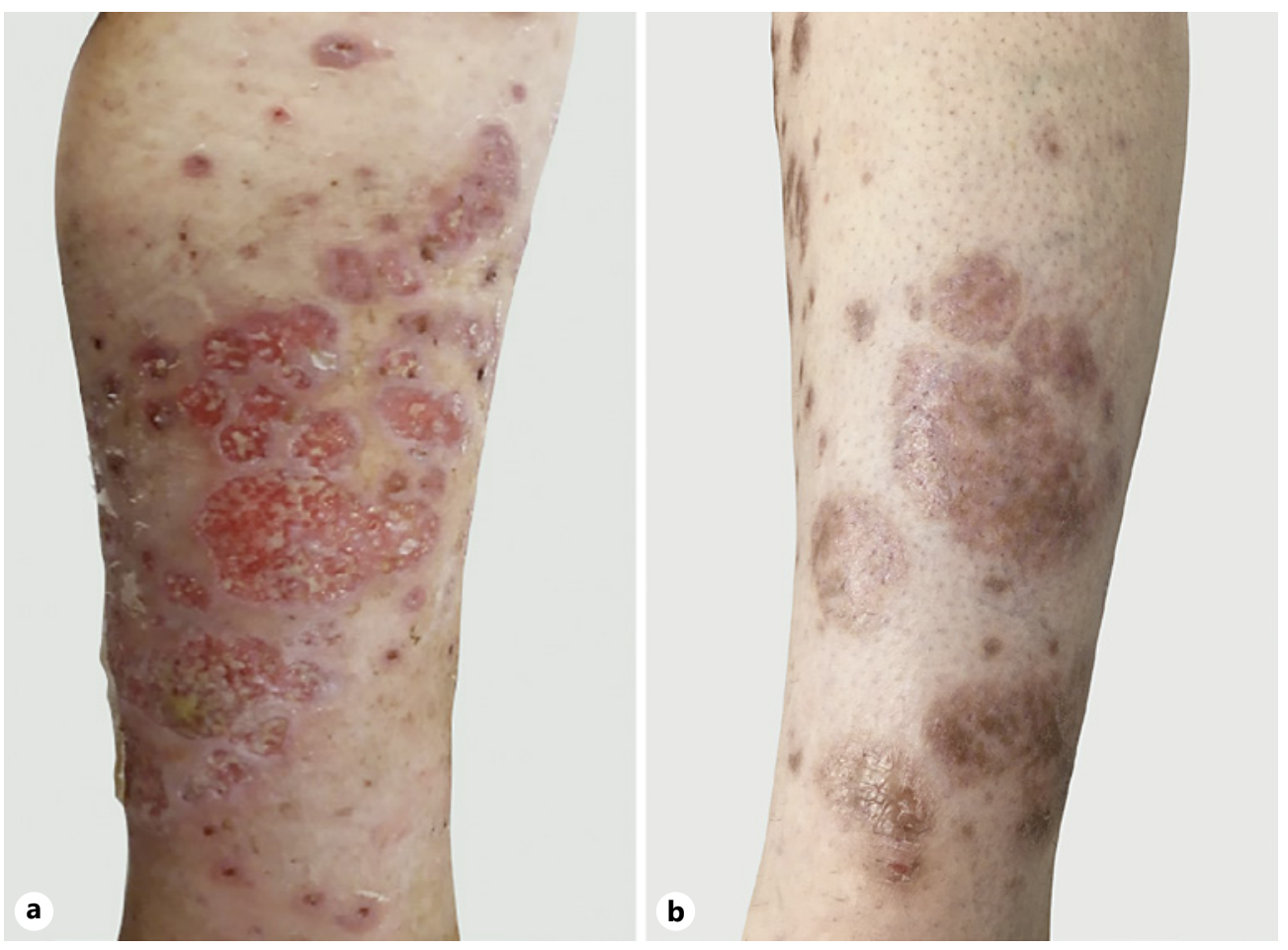

Fig. 3. Skin lesions in the healing process covered with granulation tissue (a). Patient with scars from skin lesions at the last follow-up (b).

The patient was evaluated by the dermatology team who performed a skin biopsy. The results showed a dense neutrophil population and marked dermatitis of the neutrophilic interface with dense perivascular infiltrate, fibrinoid necrosis, and leukocytoclasia suggestive of LCV (Fig. 1). The immunofluorescence for diagnosis of IgA vasculitis and systemic lupus erythematosus was negative. Rheumatologic evaluation excluded other systemic autoimmune diseases and primary vasculitis, although the patient had presented positive for antineutrophil cytoplasmic antibodies (ANCA-p) and antinuclear factor in low titers with negative autoimmune serologies. After a multidisciplinary clinical case discussion, the patient received methylprednisolone pulse therapy at $1 \mathrm{~g}$ /day intravenously for 3 days and topical therapy with ointment collagen and potassium permanganate to treat the lower extremity lesions. The patient showed significant improvement in intestinal symptoms and started prednisone at $40 \mathrm{mg}$ per day after hospital discharge. After 1 week, the patient returned to the outpatient clinic and the lesions of the lower extremities showed signs of healing (Fig. 3a). Corticosteroid tapering started and the patient began treatment with antitumor necrosis factor (TNF) therapy (infliximab at $5 \mathrm{mg} / \mathrm{kg}$ ) associated with azathioprine $(2 \mathrm{mg} / \mathrm{kg} /$ day) to focus on Crohn's disease treatment. At the last medical appointment, the patient presented no intestinal symptoms, a fecal calprotectin measurement of $44 \mathrm{mcg} / \mathrm{g}$ ( $<50 \mathrm{mcg} / \mathrm{g}$ ), and skin lesion scars on her legs (Fig. 3b).

\section{Discussion/Conclusion}

Vasculitis is an inflammation of blood vessel walls and comprises a group of rare diseases classified by the size of the affected vessels [12]. The classification includes (1) largevessel vasculitis (Takayasu arteritis and giant cell arteritis); (2) medium vessel vasculitis 
(polyarteritis nodosa and Kawasaki disease); (3) small-vessel vasculitis such as antineutrophil cytoplasmic antibody (ANCA) - associated vasculitides (granulomatosis with polyangiitis, also known as Wegener's granulomatosis), eosinophilic granulomatosis with polyangiitis (also known as Churg-Strauss syndrome), and immune complex-mediated hypersensitivity (IgA vasculitis formerly known as Henoch Schoenlein purpura); (4) variable vessel vasculitis (Behçet's disease); (5) single-organ vasculitis (cutaneous leukocytoclastic angiitis and cutaneous arteritis); (6) vasculitis associated with a systemic disease; and (7) vasculitis with probable etiologies such as hepatitis B, hepatitis C, syphilis, and drug-associated vasculitis [12].

The involvement of the gastrointestinal tract can occur in IgA vasculitis (HenochSchönlein) and Behçet's disease [12], although other vasculitis types can also. IgA vasculitis affects small vessels, predominantly capillaries, venules or arterioles, causing lesions in the skin and gastrointestinal tract in addition to arthritis [12]. Any segment of the gastrointestinal tract can be affected, but small bowel involvement is most common [12]. Behçet's disease can affect vessels of any size (small, medium, and large). It is characterized by recurrent oral and/or genital aphthous ulcers accompanied by cutaneous, ocular, articular, gastrointestinal, and/or central nervous system inflammatory lesions [12]. Differentiating between vasculitis as an extraintestinal manifestation of IBD or a systemic disease, such as those cited above, is a clinical challenge.

In the present case, the absence of systemic symptoms which would suggest primary vasculitis and the endoscopic pattern of ileum and colon lesions were evidence for the diagnosis of Crohn's disease with associated vasculitis as an extraintestinal manifestation. Furthermore, a multidisciplinary approach which included a rheumatologist and dermatologist was essential to correct diagnosis and initiation of early treatment.

A study published in 2016 [13] identified 32 patients with IBD and vasculitis, including large-vessel vasculitis ( $n=13)$, ANCA-associated vasculitis $(n=8)$, isolated cutaneous vasculitis $(n=5)$, and other types of vasculitis $(n=6)$. The data were compared to data from a literature review, in which 306 patients with IBD and vasculitis were identified, consisting of large-vessel vasculitis $(n=144)$, ANCA-associated vasculitis $(n=19)$, isolated cutaneous vasculitis $(n=66)$, LCV $(n=25)$, and other vasculitis types $(n=77)$ [13]. The patient in our case presented positive ANCA-p, which could be related to Crohn's disease, even though positivity is low in Crohn's disease compared to ulcerative colitis patients [14]. The patient had not suggestive clinical of systemic vasculitis, and the positivity of ANCA-p did not fulfill the criteria for any systemic vasculitis.

Cutaneous vasculitis is a rare extraintestinal manifestation of IBD and few reports associate it with Crohn's disease [5, 9, 11]. Lesions can appear both at the onset of symptoms and during periods of disease activity. The clinical presentation is variable, but it usually occurs with palpable purpura, urticaria, and ulcerative-necrotic lesions predominantly in the lower extremities [5,9]. The histopathological evaluation shows the presence of perivascular infiltrates rich in neutrophils, with fibrinoid necrosis of the vessel walls or the presence of granuloma $[5,9,11]$. The pathogenesis can be explained by the hypersensitivity of small vessels to circulating immune complexes [9] and since the vasculitis is related to Crohn's disease activity, there may be a similar pathogenic mechanism between them [6].

The treatment of LCV results from the appropriate treatment of IBD and the use of corticosteroids are generally recommended in the initial phase [11], as reported in this case. In a study by Sy and cols [13] which included IBD patients with any type of vasculitis, among 29 patients with information on their initial treatment for vasculitis, 93\% received corticosteroids, 79\% received immunosuppressant such as azathioprine, methotrexate, or cyclophosphamide, $21 \%$ received biologics such as infliximab, adalimumab, or abatacept, and 3\%

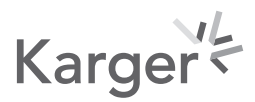


underwent plasma exchange [13]. Regarding the follow-up of the patients, $61 \%$ achieved remission. The remission rate was $56 \%$ for patients who received biological therapy $(5 / 9$ patients) [13]. TNF- $\alpha$ may play a role in the pathogenesis of different types of vasculitis, supporting the use of anti-TNF therapy in the treatment [15] Despite this, a review study concluded that, except for Behçet's disease, the use of anti-TNF has not demonstrated a significant improvement in the treatment of patients with vasculitis [15].

Another differential diagnoses that must be evaluated in IBD patients is drug-induced vasculitis, mainly induced by TNF antagonists [16]. The pathogenesis includes the formation of antibodies to TNF antagonists leading to immune complex-mediated hypersensitivity vasculitis. The lesions can also originate from the blockade of TNF- $\alpha$ pathways inducing a cytokine imbalance with a shift from a TH1 to a TH2 pattern predisposing the vasculitis onset [16]. This is not the case in the present report since the patient had not received previous treatment with anti-TNFs, but it is an important hypothesis to be considered in IBD patients under anti-TNF therapy.

IBDs are chronic and systemic diseases that can involve several organs and systems in addition to the gastrointestinal tract. Most extraintestinal manifestations occur after the diagnosis $[7,17]$ and are generally associated with disease activity $[5,18]$. However, they can appear before the diagnosis $[7,18]$, and the early recognition, the exclusion of differential diagnoses and the referral to the gastroenterologist for adequate treatment and monitoring is of paramount importance.

LCV is a rare extraintestinal manifestation that can develop in patients with Crohn's disease. Differentiating from other systemic vasculitis types is a clinical challenge. The multidisciplinary team approach is essential for the accurate diagnosis and rapid initiation of drug therapy, which are essential for disease control and restoration of the patient's quality of life.

\section{Acknowledgements}

It is not applicable for this study.

\section{Statement of Ethics}

The study was approved by the local Research Ethics Committee (Ethics Committee of Botucatu Medical School, São Paulo State University (Unesp) (CAAE: 25857119.7.0000.5411). Written informed consent was obtained from the patient for publication of this case report and any accompanying images. Written informed consent was obtained from the patient for publication of this case report and any accompanying images.

\section{Conflict of Interest Statement}

The authors have no conflicts of interest to declare.

\section{Funding Sources}

This research did not receive any specific grant from funding agencies in the public, commercial, or not-for-profit sectors.

\section{Karger's}




\section{Author Contributions}

All authors contributed to this manuscript. Thiara Barcelos Rocha, Ana Lorena Sousa de Vasconcelos Garate, Rodrigo Fedatto Beraldo, Sean Hideo Shirata Lanças, Fábio Vicente Leite, Jaqueline Ribeiro de Barros, Julio Pinheiro Baima, Rogerio Saad-Hossne, and Ligia Yukie Sassaki contributed to the conception and design of the study; the acquisition, analysis, and interpretation of data; drafting the article, revising it critically for important intellectual content and approving the final version to be submitted. Rodrigo Quera had substantial contributions to interpretation of data, revising it critically for important intellectual content and final approval of the version.

\section{Data Availability Statement}

The datasets used and/or analysed during the current study are available from the corresponding author on reasonable request.

\section{References}

1 Veloso FT. Extraintestinal manifestations of inflammatory bowel disease: do they influence treatment and outcome? World J Gastroenterol. 2011;17(22):2702-7.17

2 Ko JS, Uberti G, Napekoski K, Patil DT, Billings SD. Cutaneous manifestations in infammatory bowel disease: a single institutional study of non-neoplastic biopsies over 13 years. J Cutan Pathol. 2016;43(11):946-55.

3 Greuter T, Navarini A, Vavricka SR. Skin manifestations of inflammatory bowel disease. Clin Rev Allergy Immunol. 2017;53(3):413-27.

4 Gionchetti P, Dignass A, Danese S, Magro Dias FJ, Rogler G, Lakatos PL, et al. European evidence-based consensus on the diagnosis and management of Crohn's disease 2016: part 2: surgical management and special situations. J Crohns Colitis. 2017;11(2):135-49.

5 Marzano AV, Borghi A, Stadnicki A, Crosti C, Cugno M. Cutaneous manifestations in patients with inflammatory bowel diseases: pathophysiology, clinical features, and therapy. Inflamm Bowel Dis. 2014;20(1): 213-27.

6 Gravina AG, Federico A, Ruocco E, Lo Schiavo A, Romano F, Miranda A, et al. Crohn's disease and skin. United Eur Gastroenterol J. 2016;4(2):165-71.

7 Vavricka SR, Schoepfer A, Scharl M, Lakatos PL, Navarini A, Rogler G. Extraintestinal manifestations of inflammatory bowel disease. Inflamm Bowel Dis. 2015;21(8):1982-92.

8 Lakatos PL, Lakatos L, Kiss LS, Peyrin-Biroulet L, Schoepfer A, Vavricka S. Treatment of extraintestinal manifestations in inflammatory bowel disease. Digestion. 2012;86 Suppl 1(1):28-35.

9 Huang BL, Chandra S, Shih DQ. Skin manifestations of inflammatory bowel disease. Front Physiol. 2012;3:13.

10 Brown SR, Coviello LC. Extraintestinal manifestations associated with inflammatory bowel disease. Surg Clin North Am. 2015;95(6):1245-59, vii.

11 Hagen JW, Swoger JM, Grandinetti LM. Cutaneous manifestations of Crohn disease. Dermatol Clin. 2015;33(3): 417-31.

12 Jennette JC, Falk RJ, Bacon PA, Basu N, Cid MC, Ferrario F, et al. 2012 revised International Chapel Hill consensus conference nomenclature of vasculitides. Arthritis Rheum. 2013;65:1-11.

13 Sy A, Khalidi N, Dehghan N, Barra L, Carette S, Cuthbertson D, et al. Vasculitis in patients with inflammatory bowel diseases: a study of 32 patients and systematic review of the literature. Semin Arthritis Rheum. 2016; 45(4):475-82.

14 Zhou G, Song Y, Yang W, Guo Y, Fang L, Chen Y, et al. ASCA, ANCA, ALCA and many more: are they useful in the diagnosis of inflammatory bowel disease? Dig Dis. 2016;34(1-2):90-7.

15 Jarrot PA, Kaplanski G. Anti-TNF-alpha therapy and systemic vasculitis. Mediators Inflamm. 2014;2014: 493593.

16 Hindryckx P, Novak G, Costanzo A, Danese S. Disease-related and drug-induced skin manifestations in inflammatory bowel disease. Expert Rev Gastroenterol Hepatol. 2017;11:203-14.

17 Harbord M, Annese V, Vavricka SR, Allez M, Barreiro-de Acosta M, Boberg KM, et al. The first European evidence-based consensus on extra-intestinal manifestations in inflammatory bowel disease. J Crohns Colitis. 2016;10(3):239-54.

18 Vavricka SR, Scharl M, Gubler M, Rogler G. Biologics for extraintestinal manifestations of IBD. Curr Drug Targets. 2014;15(11):1064-73. 